\title{
Answer to the Letter to the Editor of O. Ersen concerning "Strap stabilization for proximal junctional kyphosis prevention in instrumented posterior spinal fusion" by Rodriguez-Fontan F, et al. (Eur Spine J; [2020]:1287-1296, https://doi.org/10.1007/ s00586-020-06291-0)
}

\author{
Francisco Rodriguez-Fontan ${ }^{1,2,3} \cdot$ Bradley J. Reeves $^{1,2}$ - Andriy Noshchenko ${ }^{1}$. David Ou-Yang ${ }^{1} \cdot$ Christopher J. Kleck $^{1}$. \\ Christopher Cain ${ }^{1}$. Evalina Burger-Van der Walt ${ }^{1}$. Vikas V. Patel ${ }^{1}$
}

Received: 17 July 2020 / Accepted: 17 July 2020 / Published online: 4 August 2020

c) Springer-Verlag GmbH Germany, part of Springer Nature 2020

Thank you for your interesting comments to our study. Mersilene tape is non-absorbable and has minimal tissue reactivity; hence, whatever tension it gets in vivo is technically preserved and doesn't decrease over time. Viswanathan et al. show an interesting biomechanical comparative study using Mersilene tape and sublaminar bands. Although significant, whether clinically relevant, yet, has to be tested.

In our study, Mersilene tape knots were tighten by hand, aiming to restore the integrity of the posterior tension band which is compromised by exposure of the posterior elements. The tape is not elastic, and knots should be tight. The alignment of the vertebrae should be physiological, and the band to be felt tense. Compression of the posterior elements or laxity is unacceptable. The tension is not directly measured or limited mechanically. As stated above, the tightness of the tape is expected to be preserved.

Regarding failure time, the patients treated with Mersilene tape had a significant delayed presentation for PJK/PJF, averaging 20 months. The level above the transition zone of
PSF + Mersilene tape was the most affected level. The failure was through the soft tissue or the osseous elements, not from Mersilene tape failure.

Whether proximal junctional measurements can give information of the tightness of the tape or if the length, and hence, the tape tightness can be measured with ultrasound in order to predict who is at higher risk of progression, are future questions to be explored.

\section{Compliance with ethical standards}

Conflict of interest The authors declare that they have no conflict of interest.

Publisher's Note Springer Nature remains neutral with regard to jurisdictional claims in published maps and institutional affiliations.

Francisco Rodriguez-Fontan

francisco.rodriguezfontan@cuanschutz.edu

1 Department of Orthopedics, University of Colorado Anschutz Medical Campus, 12631 East 17th Ave, Aurora, $\mathrm{CO}$, USA

2 School of Medicine, University of Colorado, Anschutz Medical Campus, 13001 E 17th Pl, Aurora, CO, USA

3 Facultad de Medicina, Universidad de Buenos Aires, Paraguay 2155, 10109 CABA, Buenos Aires, Argentina 